$\xi=$

\title{
Systematic Cluster Head (CH) Selection Through Node-Grade Based Clustering (NGBC) in WSN
}

\author{
J.K. Deepak Keynes ${ }^{1 *}$, D. Shalini Punithavathani ${ }^{2}$ \\ ${ }^{1}$ Research Scholar, Department of Computer Science \& Engineering, Manonmaniam Sundaranar University, Tirunelveli, India. \\ ${ }^{2}$ Former Principal, Government College of Engineering, Tirunelveli, India. \\ E-mail:shalini329@gmail.com \\ *Corresponding author E-mail:deepak.keynes@gmail.com
}

\begin{abstract}
As it is well known, in Wireless Sensor Networks, the sensor nodes will be either mobile or static. When mobility is concerned, on the whole network performance could be degraded, since the sensor nodes are furnished with restricted battery power, restricted memory, less computational ability and lower range of communication. So, a mechanism which is effective is needed there for forwarding the data packets with efficient energy management and coverage. With that note, the principle target of this work is to propose systematic method of $\mathrm{CH}$ selection based on the factors such as low mobility, density of the nodes and their remaining energy. Moreover, an innovative method called Node-Grade Based Clustering (NGBC) is proposed in this paper so as to select the CHs, studying the node's energy and position regarding to their Base Station (BS), which will act as a sink for collected information. The CHs are replaced in every round based on its duty cycle on sensor nodes and Threshold Energy Rate (TER). Since the BS evaluates the quantity of every round a CH (Cluster Head) can sustain, it minimizes the quantity of energy consumed and increases the WSN's lifetime. The results of the simulation demonstrate that the proposed algorithm attains higher coverage, efficiency in energy and network lifetime. Furthermore, the performance results in the work which is proposed, are distinguished with the algorithms proposed previously such as LEACH and HEED using some evaluation metrics like packet delivery ratio, throughput, energy consumption and end-to-end delay to prove the efficiency of energy efficient NGBC.
\end{abstract}

Keywords: Wireless Sensor Networks (WSN), Cluster Head (CH), Sensor Nodes, Node- Grade Based Clustering (NGBC), network longevity, residual energy.

\section{Introduction}

Wireless Sensor Networks (WSN) comprises group of static or mobile nodes those are suitable of communicating each other and transfer data effectively and autonomously. Moreover, WSN can be providing some additional benefits and adaptability in low power, cost effectiveness and fast deployment respectively in several applications when there's no requirement of human management or supervision. Some of the vital WSN's applications are found in various fields such as wild life tracing, health care observation, rescue operations and military applications. In general, the deployed sensor nodes monitors the ecological or physical occurrences like temperature changes, vibration, pressure, movements, sounds, pollutants or some typical activities at various locations.

The distributed sensor nodes are needed to send the detected information to the BS in continuous manner. Because of limited energy in all sensor nodes, so many approaches had been created to adjust the energy accumulation in WSN in effective manner. The overall energy in sensor nodes is basically consumed by four units, namely, sensing unit, power unit, computing unit and communication unit. The figure 1 depicts the major components present in every sensor node. Additionally, the sensing unit comprises of sensors to Analog-to-Digital Convertors (ADC) where the observed analog signals are changed over to digital and sent to computing unit. The computing unit encompasses of a small storage which involves sensor nodes collaborations. Then, the transceiver is to ensure the correspondence between the network and the nodes. As is well known, the sensor nodes has limited power, there includes a power unit equipped with some limited source for usage.

Location finding system is to have the better knowledge about location accuracy. Mobilizer is to give mobility factor of nodes on the network.

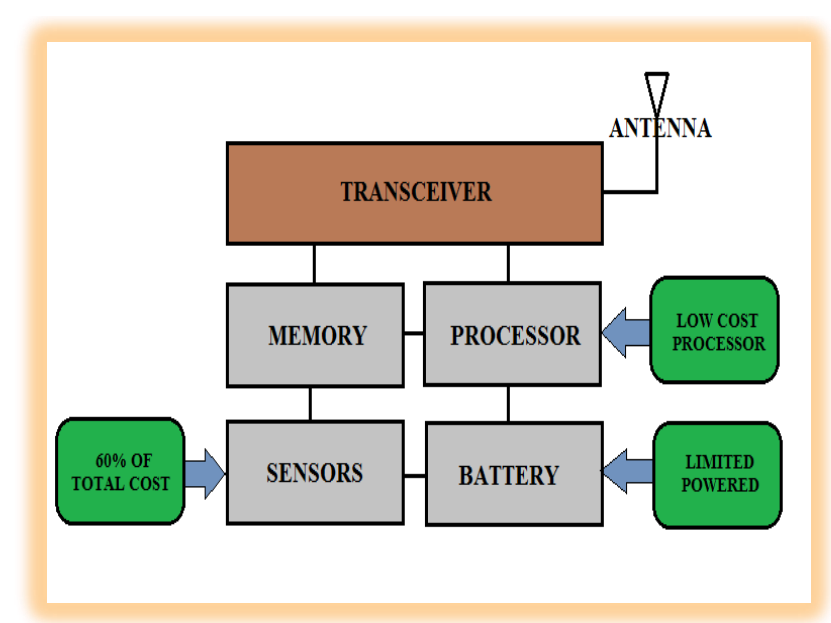

Figure 1: Major segments of every sensor node 
Figure 2 presents the main architecture of cluster based WSN. It is clearly stated from the figure that the BS collects the information sent from the $\mathrm{CHs}$ of the distributed sensor nodes.

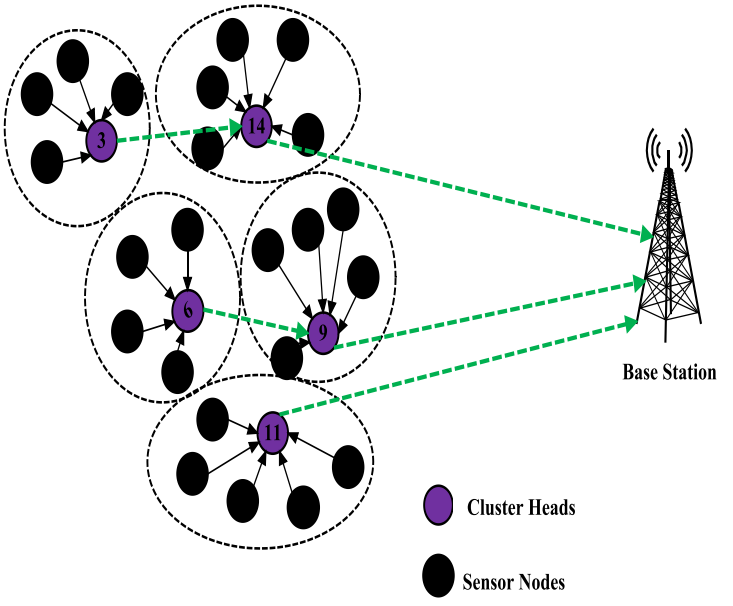

Figure 2: Architecture Basic of Cluster Based $[\mathrm{CH}]$ in WSN

In WSN, clustering is a significant technique for cost reduction in data collection process. According to that, the sensor nodes are combined into disjoint groups and each group is managed the Cluster Head, which is elected among the sensor nodes. The resource allocation and simultaneously energy consumption into every node is also been reduced by the cluster head $[\mathrm{CH}]$ sensing process in WSN. Hence, the network's longevity is also been increased [2]. As mentioned before, $\mathrm{CH}$ controls every sensor nodes in an appropriate cluster and those $\mathrm{CHs}$ have their capability of transferring information with the Base Station [BS] directly. The rest of the nodes are insisted to deliver their data which is sensed, to the corresponding $\mathrm{CH}$. Then, the work of $\mathrm{CH}$ is to collect and combine those data and forwarding that to the BS. Due to these significant tasks performed by $\mathrm{CHs}$, effective cluster, specifically, formation best possible $\mathrm{CH}$ selection has become the important domain for research in WSN and it also affects the network considerably.

It is to be stated that, while a clustering protocol is designed, the size of the cluster along with ensuring connectivity throughout the network's lifetime are to be considered. The sensors are to be allowed to sleep, when there no requirement of its active contribution, which increases the network's longevity. Therefore, the work cycle of every node has also to be studied while a clustering mechanism is designed. With all those concerns, this paper introduces a mechanism called Node-Grade Based Clustering (NGBC) algorithm. Every cluster's sensor nodes are allotted with grades on its current energy levels and distance between the Euclidean distance and the data sink or the BS and the nodes. In general, the design of every CH's selection process involved in selecting the $\mathrm{CH}$ based upon the nodes having highest residual energy. That may causes some inefficiency like a node may take longer way in communicating with the BS, energy would be consumed more while replacing the $\mathrm{CHs}$ in each rounds. The algorithm proposes solution for these problems by directing the exact path to BS, using energy threshold for computing the remaining energy of every sensor node effectively. Hence, the results outperform some previous methodologies and provide better solution.

The remaining sections of this paper are systematized as follows: Section II gives a reflection on the study of related works. Section III provides a description about various issues to be noted on developing clustering algorithm in WSN. Section IV gives out the detailed work process of the newly proposed NGBC algorithm for effective $\mathrm{CH}$ selection. The experimental results and comparison charts are provided in section V. Lastly, the paper concludes with section VI with some pointers to future work.

\section{Related works}

There are numerous approaches have been proposed for designing a cluster based Wireless Sensor Network (WSN). Probably, Low Energy Adaptive Clustering Hierarchy (LEACH) [3], the first routing protocol based upon clustering methodology in WSN, uses a speculative model for electing a $\mathrm{CH}$ and a motivation for much other architecture which is similar to LEACH. There is an assumption that there occurs a distinctive base station exterior to the sensor network coverage area and a direct manner of communication with the BS happens in each of the sensor nodes inside the network. For saving more energy, the LEACH model involves in choosing a $\mathrm{CH}$. The nodes those are remaining are grouped as clusters in light of its signal strength from $\mathrm{CHs}$ and the operations of nodes are described as rounds. After forming clusters, in every round, the $\mathrm{CHs}$ process the data gathering that are collected from the normal cluster members and thereafter send that to the respective BS through single hop communication.

In [14], the aforementioned approach was handled in a marginally extraordinary way and named as LEACH-C. The approached are processed with the even distribution of $\mathrm{CHs}$ all throughout the network and limiting energy dissemination. At the beginning, every node is needed to provide data about its level of energy and location to the respective BS. Then, the BS executes an efficient methodology for establishing the cluster formation for that round. Hence, the LEACH-C model requires a Global Positioning System (GPS) for this case. In another way, in [10], the selection of the $\mathrm{CHs}$ depends upon the node's remaining energy that makes the decision if the normal node becomes $\mathrm{CH}$ or not after completion of the first round. E-LEACH is the approach named there, that elects the node having higher energy as the $\mathrm{CH}$ and considers the remaining nodes having lesser energy as cluster heads. Sajjanhar et al. [1] developed a Distributive Energy Efficient Adaptive Clustering (DEEAC) protocol for effective clustering in networks having Spatio-temporal differentiations in data reporting ratio in different locations. The $\mathrm{CH}$ selection was performed in view of hotness value and the present energy level of the sensor nodes.

VLEACH [15] is a different clustering protocol that is developed on focusing the issue of $\mathrm{CH}$ which dies before the members inside the clusters. During such cases, in VLEACH, there exists a ViceCluster Head along with the elected $\mathrm{CH}$ for acting as $\mathrm{CH}$. So, the $\mathrm{CHs}$ are always reachable to $\mathrm{BS}$ and there becomes no necessity of new $\mathrm{CH}$ selection.

In WSN, network longevity is a necessary factor to be studied while developing a clustering protocol. With that concern, Maximize Network Lifetime Protocol (MNLP) [11] has been developed for adjusting with heterogeneous WSN. The process involved in selecting $\mathrm{S}_{\text {Head }}$ called the set of $\mathrm{CH}$ as per the measurements like the data about the present energy level of a particular node with the mean value of remaining energy of its neighbour nodes. Zytoune et al. [4] inherited the LEACH algorithm after modifying the possibility of every sensor node inside the cluster to turn $\mathrm{CH}$ in view of its energy requirement in transmitting the data collected to the sink. An effective algorithm for clustering in view of Ant-Bee agent is described in [17].

Mixed Non-Uniform Clustering algorithm (MNUC) has been proposed by Du et al. [5] which increases the WSN lifetime. The work mainly concentrated on the hot spot issues where the sensors are needed to be more active while comparing with the other regions of network coverage. That is, more energy would be consumed in those cases than the others. So, the aim of the algorithm is to form unequal clusters. Forming smaller clusters with the node that are very near to the sink and to increase the cluster sizes, when it becomes larger distance than the BS. Therefore, the smaller sized clusters are neared to the respective BS and utilized more, but, transmission range will be low. In [13], Universal LEACH denoted as ULEACH has been framed as an extension of LEACH. As is well known, CHs are picked upon the basis of initial and remaining energy in every sensor nodes. 
Moreover, it performed multi hop information transfer over the network. Another methodology called T-LEACH [6] that improves the LEACH by performing threshold based $\mathrm{CH}$ selection. It also uses the random $\mathrm{CH}$ selection without following any special mentioned criteria. IDCA [16] (Integrated Distributed Clustering Algorithm) is a recent approach developed for optimal clustering in WSN. The process is based upon $\mathrm{CH}$ selection, aggregating data and forwarding to $\mathrm{BS}$ through $\mathrm{CHs}$ and also maintains an emergency prevention mode to handle clusters in critical cases.

Hybrid Energy Efficient Distributed (HEED) [12] clustering protocol involves two parameters on selecting cluster heads, node's closeness to its neighbourly nodes and node's residual energy. It assures for systematic $\mathrm{CH}$ distribution over the whole network. Inter and intra-cluster communications are considered during designing the protocol. All the above algorithms will prolong network longevity and balances the workload among the distributed sensor nodes using some factors for clustering, the maintenance and selection of $\mathrm{CH}$.

\section{Major issues on clustering algorithm}

As indicated by the related works described in the previous section (Section 2), there are number of issues are to be considered while developing a clustering algorithm for large scale wireless sensor networks. Those challenges are narrated below.

\subsection{Cluster size}

There is an assumption in most of the existing clustering approaches which all the clusters are equal in physical size. This may direct to unbalanced load where the cluster heads [CHs] that is neared with the sink dissipates higher energy than the far-away CHs. In [7], Shu et al. suggested that a larger cluster is needed by the $\mathrm{CHs}$ which are having minimal data to send. However, the nodes are required to know their located area in addition to the BS. Therefore, appropriated cluster sizes are to be chosen and the energy consumption in WSN is decreased, in view of the parameters, such as communication range, node density, and locations of the BS, and also the frequencies of data aggregation.

\subsection{Connectivity maintenance}

One among the significant objectives of clustering protocols is making network connectivity to be guaranteed. It is to be maintained that every last node in the network ought to be associated with a cluster. It's also to be kept that the whole nodes in a specific cluster must be able to convey data with its $\mathrm{CH}$ for avoiding multi hoping problems and reducing energy consumption. For solving connectivity related issues on WSN, in [9], the authors have pointed that the nodes presented on cluster borders are utilized as gateways for dispatching the information among CHs. Additionally, the network's proximity is needed to be sufficient in ensuring that the required gateways are located at the overlapping areas between formed clusters. In a distinct way, as per the authors of [13 and12], predominant selection of intercluster and intra-cluster communication ranges to connectivity maintenance and prolonging the network's lifetime, a significant task to be observed during development of a clustering algorithm.

\subsection{Clustering based on duty-cycle}

When the sensor nodes aren't required to provide its active contribution, it is to be allowed to stay on sleep mode which helps in the lifetime of the node extended. Hence, duty cycle of each of the node in the cluster ought to be considered on designing clustering algorithms. Duty cycles of nodes are incorporated in the designing process in view of the following two cases based on the application.
CASE 1: the non - $\mathrm{CH}$ nodes will be requested to become on sleep mode, when they aren't included in data sensing work or not communication with its $\mathrm{CH}$.

CASE 2: when the applications need the nodes to constantly monitor the location, then the $\mathrm{CH}$ can find the cluster members who are sending redundant information and make them to sleep [8].

\subsection{Cluster head selection}

This is an even more important issue in WSN as discussed earlier. After the arrangement of the clusters, the next vital task is to pick the best cluster head $[\mathrm{CH}]$ for each divided cluster. The selected $\mathrm{CH}$ should be reachable for each non- $\mathrm{CH}$ nodes and should also be involved in increase of the lifetime and adaptability of whole network. Many ways are proposed for effective $\mathrm{CH}$ selection in view of having high current energy among others or could be approached by all members with lesser energy. Further, it is also vital to shift the CH's role among all members to evade overloading some nodes with even more responsibilities and consumers its energy more. The review of excellent $\mathrm{CH}$ selection and rotating responsibilities are more fundamental for increasing the network's longevity and accountability [6].

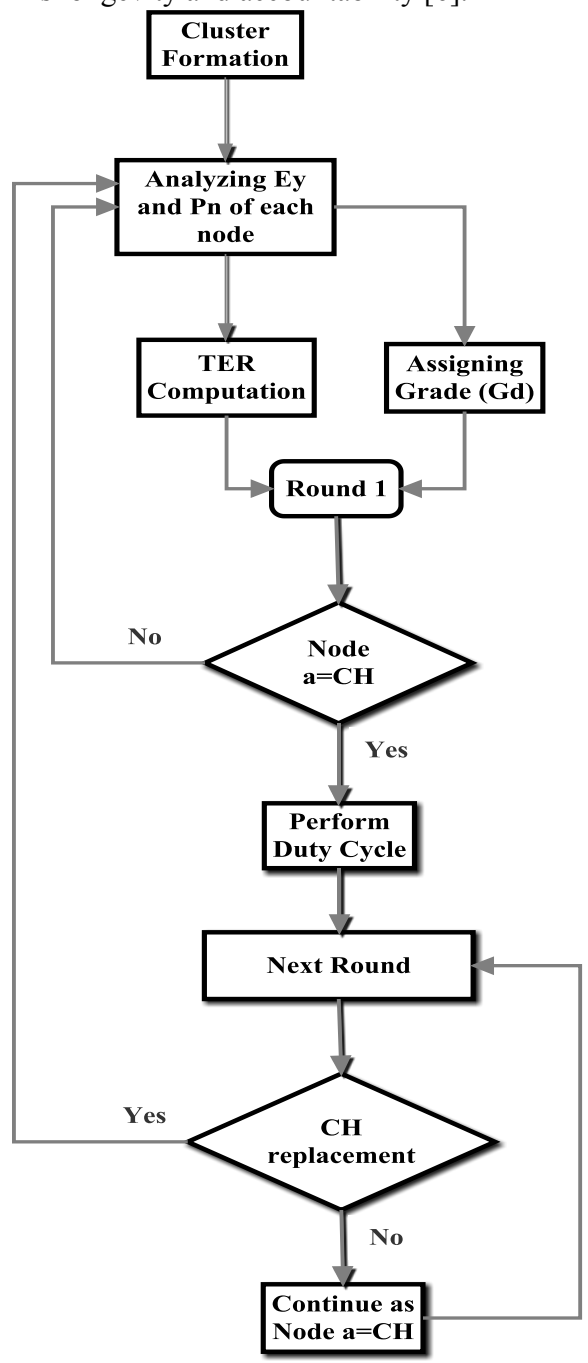

Figure 3: Work-flow of proposed NGBC Algorithm

\section{Node - Grade based Clustering (NGBC) algorithm}

By concluding the above discussions, this paper comes up with a new algorithm called Node-Grade Based Clustering (NGBC) algorithm for selecting excellent $\mathrm{CHs}$ in WSN. The mechanism 
helps in extending the network's lifetime while reducing the communicational overheads due to the frequent $\mathrm{CH}$ selection that tends to raise the consumption of energy on nodes than other approaches. It can be attained by appropriate selection and $\mathrm{CH}$ replacement based on current energy levels of sensor nodes and the distances measured between the respective nodes. The algorithm also incorporates the energy threshold utilization and evaluation of number of rounds that the particular $\mathrm{CH}$ is required to be serving before its getting replaced. Furthermore, in this approach, the nodes inside the cluster are allotted with grade points in view of present energy level (Ey) and its position (Pn) with respect to the BS. This grading system is used for selecting efficient $\mathrm{CHs}$ which are sorted into levels in accordance to its Euclidean distance and its position from the respective sink. Hence, every nodes in the networks are assigned with a grade, denoted as Gd (Ey,Pn), pointing its probability for being selected as a $\mathrm{CH}$.

Additionally, by using energy threshold techniques in decision making for effective $\mathrm{CH}$ replacement improves the nodes' lifetime which are nearby to the base station [BS]. From that, it is obvious, the complete network's lifetime is then increased in an effective manner, specifically, and connectivity towards sink becomes stronger. In the proposed algorithm, it is pointed out that the BS is in static position and reserves infinite energy. Hence, there are no limitations considered on power or energy consumption during the overall data processing and network communication. The steps which are to be considered for designing the NGBC algorithm are the following

- The base station divides the area covered into smaller portions which is termed as clusters, in accordance to the predefined communication ranges of the sensor nodes and its positions.

- It has to be assured that no nodes are out of coverage area.

- In set-up phase, every nodes broadcast a message to its neighbourly nodes about its present energy level and location.

- Hence, every node maintains a data table about its neighbours that is again advertised to their neighbourly nodes til the data about all of the sensor nodes are reached to sink.

- The BS evaluates the number of rounds every $\mathrm{CH}$ can be serving in its position in accordance with its remaining energy and threshold energy value, which has been giving the data to every cluster head $[\mathrm{CH}]$.

- The $\mathrm{CHs}$ closer to the BS will have larger energy threshold rate, whereas, farer $\mathrm{CHs}$ will have smaller energy threshold rate.

- The CHs should be displaced only when their remaining energy level becomes lesser than the predefined threshold rate.

- If there is any alteration in the topology of the network, when the nodes attain lower residual energy than threshold or dead, it's the responsibility of the BS to establish another suitable $\mathrm{CH}$ for every cluster respectively.

With those assumptions, the pseudo code for NGBC algorithm is shown below. Figure 3 represents the flow chart of the proposed Node-Grade Based Clustering Algorithm for efficient and effective $\mathrm{CH}$ selection in WSN.

\subsection{NGBC algorithm}

\section{ifClustered_network $=$ false}

for node $\mathrm{n} \mathrm{N}$ (Node_list) do

$\mathrm{n}$ broadcast their energy level and position to the base station

for every node a N (Node_list) do

sorting nodes based on geographical location

// nodes are aggregated in view of its range of communication

if distance between node a and a+1 <range_of_communication

add $\mathrm{a}$ and $\mathrm{a}+1$ to Clsuter_list $\mathrm{C}_{1}$

else

Created new Cluster_list $\mathrm{C}_{2}$

add $\mathrm{a}+1$

end if

$\mathrm{a}+1$

end for

for every node $\mathrm{n} \quad \mathrm{N}$ (Node list) do

grade (n)=BS assigns grade to ' $\mathrm{n}$ ' based on its current energy level (Ey) and Euclidean position from the base station[BS]

end for

for every node $\mathrm{n} \quad \mathrm{N}$ (Node_list) same location do

if $(\operatorname{grade}(\mathrm{n})>\operatorname{grade}(\mathrm{n}+1))$ then

can_become Cluster Head $(\mathrm{CH})=$ true

add node to $\mathrm{CH}$ Candidate list $\mathrm{CH}$ CL

end if

$n+1$

end for

for each $\mathrm{CH}$-C CH_Candidate_list do

$\mathrm{CH}$ candidates are graded into levels in accordance to their position from BS

end for

for every node $\mathrm{CH}$ ClusterHeads_list do

evaluate number of rounds $\mathrm{CH} \_\mathrm{C}$ can be as Cluster Head

$\mathrm{n}$ combines the $\mathrm{CH}$

\subsection{Evaluation for optimal $\mathrm{CH}$ selection}

Following cluster formation, a $\mathrm{CH}$ will be assigned for each cluster by the BS on the affirmed NGBC algorithm. The sensor nodes in every cluster are assigned with grade points in accordance to their corresponding distance from the BS and its current energy level. Taking the following equations (1), (2) and (3), the nodes which are having higher remaining energy and lesser Euclidean distance will be picked as a $\mathrm{CH}$.

Grade Gd $(E y, P n)$

$$
\begin{aligned}
& \operatorname{Pn}(a)=\operatorname{Minimum}(\operatorname{Dist}(a, b s)) \\
& (\operatorname{Ey}(a))=\operatorname{Maximum}(\text { Residual Energy })
\end{aligned}
$$

$|\operatorname{Dist}(a, b s)|=\sqrt{\left(X_{a}-X_{b s}\right)^{2}+\left(Y_{a}-Y_{b s}\right)^{2}}$

From the above equations, Ey denotes the present energy level or remaining energy of the node i, Dist $(a, b s)$ points the Euclidean distance between the particular node ' $a$ ' and the base station. It is also shown in the equation about the specific exploitation of region of interest, the axial positions, $X_{a}$ and $Y_{a}$ are the positions of node ' $\mathrm{a}$ ' in accordance to its $\mathrm{X}$ and $\mathrm{Y}$ axis. $\mathrm{X}_{\mathrm{bs}}$ and $\mathrm{Y}_{\mathrm{bs}}$ represent the locations of BS. As per the algorithm, the $\mathrm{CH}$ in every cluster are to be replaced with another node when its level of energy drops under a calculated Threshold Energy Rate (TER). That is, it is possible for a member ' $a$ ' of a cluster to be a $\mathrm{CH}$ in single or multiple rounds of communication. The TER of every node is computed in view of the following equations (4), (5) and (6).

Threshold Energy RateTER $(a)=\frac{\operatorname{Residual}(E y(a))}{\operatorname{Average}(E y)} \times \frac{\operatorname{Average}(P n)}{\operatorname{Dist}(a, b s)}$ 


$$
\begin{aligned}
& \operatorname{Average}(P n)=\frac{\sum_{a=1}^{n} \operatorname{Dist}(a, b s)}{n} \\
& \operatorname{Average}(E y)=\frac{\sum_{a=1}^{n} \operatorname{Residual}(\operatorname{Ey}(a))}{n}
\end{aligned}
$$

The above mentioned equations give the computational results for Threshold Energy Rate of the total nodes in the cluster. Obviously, in the first round, all of the sensor nodes in the networks contain same energy levels. As a result, the grading process will be depending only upon the Euclidean distance. The nodes closer to the BS have the better probability on becoming the $\mathrm{CH}$, later on, the remaining energy of every node will vary. Based upon the equation (4), nodes which are very nearer to the base station will be replaced faster, since it contains higher TER. This is also because, those closer nodes are important for aggregated data to the BS. In other way, nodes which are away from the sink will comprise lower TER and will not be replaced often. The number of rounds a node ' $\mathrm{a}$ ' can sustain as $\mathrm{CH}$ is calculated using the equation (7). The evaluation is calculated based on the remaining energy of the node and the computed TER. The equation is given as follows,

$$
N u m_{-} \text {of_rounds }(a)=\frac{\operatorname{Residual}(\operatorname{Ey}(a))}{\operatorname{TER}(a)}
$$

Based on that, the $\mathrm{CH}$ should be replaced in every round to save the node's energy from additional or wasted consumption.

\section{Performance evaluation and discussions}

To provide evidence to the efficiency of proposed mechanism, the NGBC is compared with the results obtain from previous algorithms like LEACH, HEED and IDCA. The comparison has been done in view of metrics such as energy consumption, throughput, packet delivery ratio, end-to-end delay and packet drop. Moreover, the performance of the affirmed work is evaluated using Network Simulator tool (NS2) with the initial parameter assumptions as in table 1. The simulation area taken here is $500 \mathrm{~m} \times 500 \mathrm{~m}$ and every nodes are assigned with the starting energy 5 Joules and it has to be taken into consideration that the data accumulation process consumes $5 \mathrm{~nJ}$ per bit.

Table I: Simulation Parameters with Initial Settings

\begin{tabular}{|c|c|}
\hline Parameters & Initial Values \\
\hline Simulator & Ns-2.34 \\
\hline Sensing Area & $500 \mathrm{~m} \mathrm{X} \mathrm{500m}$ \\
\hline Simulation Time & Varies From $1-100$ \\
\hline No. Of Nodes & 50.0 \\
\hline Simulation End Time & Random Waypoint \\
\hline Mobility Model & Ieee 802.11 \\
\hline Mac Type & Cbr \\
\hline Traffic Type & $5 \mathrm{M} / \mathrm{S}$ \\
\hline Mobility Speed & $0 \mathrm{~S}$ \\
\hline Pause Time & $512 \mathrm{Bytes}$ \\
\hline Payload Size & $250 \mathrm{M}$ \\
\hline Transmission Range & 5 joules Per Node \\
\hline Initial Energy & $9 \mathrm{Mhz}$ \\
\hline Frequency & Based
\end{tabular}

The formation of cluster in Node-Grade Based Clustering (NGBC) Algorithm is shown in the following figure 4. As per the algorithm, the nodes which are nearer to the BS are taken as the $\mathrm{CHs}$ in every cluster.

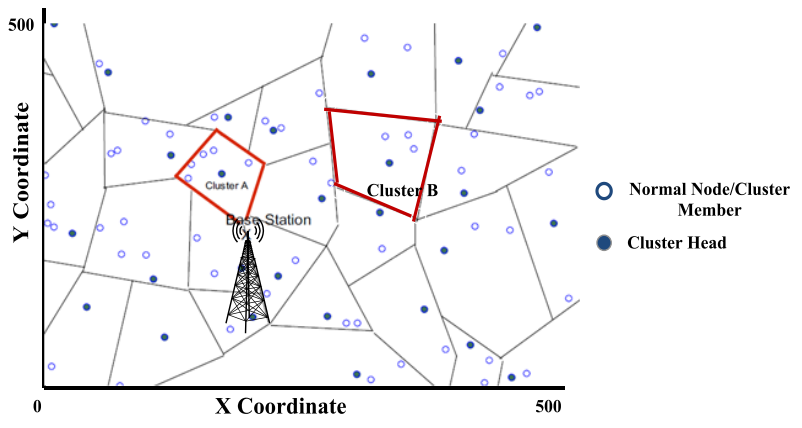

Figure 4: NGBC cluster formation and $\mathrm{CH}$ selection

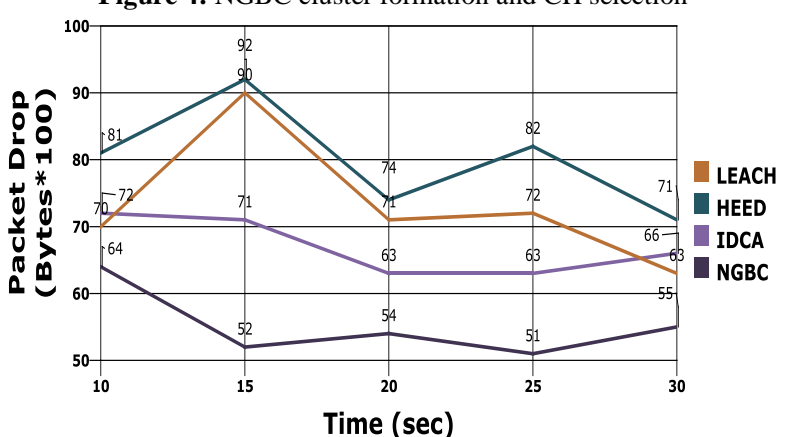

Figure 5: Packet drop comparison

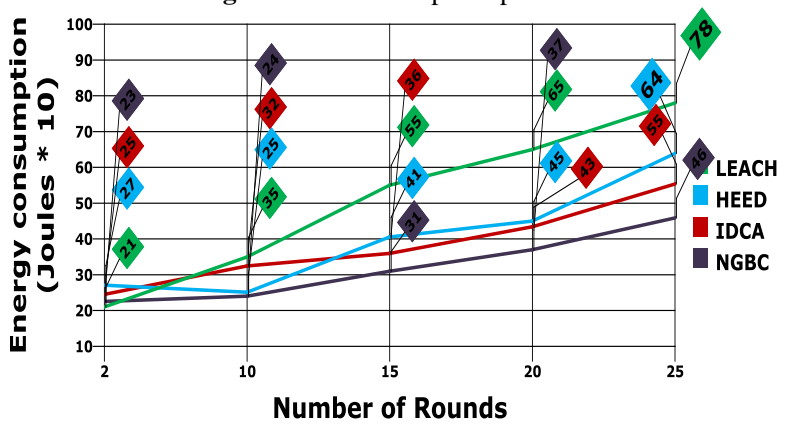

Figure 6: Energy consumption Vs number of rounds

The graph shown in figure 5 compares the packet lost by LEACH, HEED, IDCA and NGBC with correspondence to time during the overall sensing communication in WSN. Packet loss takes place when the data packets moving above the network declined to reach the target. Perhaps, it may cause due to the network's congestion. It is computed as the percentage of data packets dropped in relation to the sent packets. The above graph shows that the proposed approach provides lesser packets dropped on compared with others.

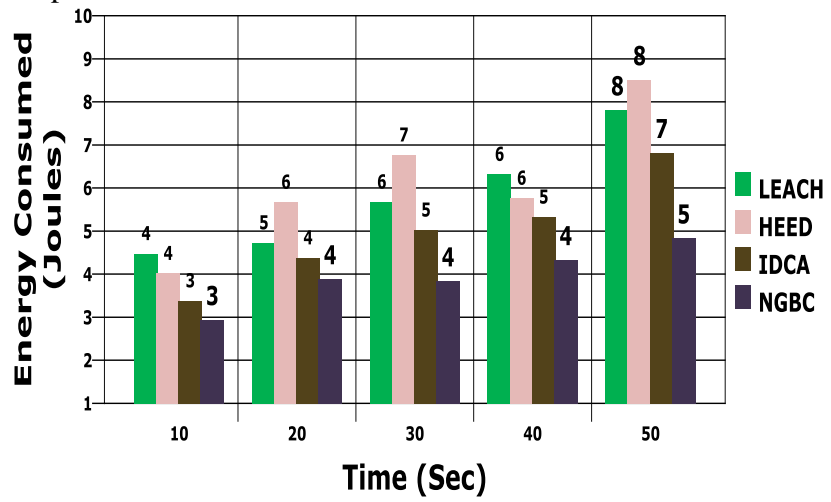

Figure 7: Energy consumption Vs time

Following that, the energy consumption rate of the algorithm is evaluated as the ratio of total energy consumed and the number of data packets received. Here, energy consumption is analyzed based on number of rounds and the time. The results are shown in the figure 5 and figure 6 . From the figure 5 , at the end of 25 
rounds, the NGBC algorithm consumed 4.6 joules of energy that is comparatively lower than the others. And also, from figure 6 , the consumption of energy by the nodes in the proposed method of clustering is considerably low. Hence, it has better remaining energy for providing active and effective communication, and increases the network's lifetime.

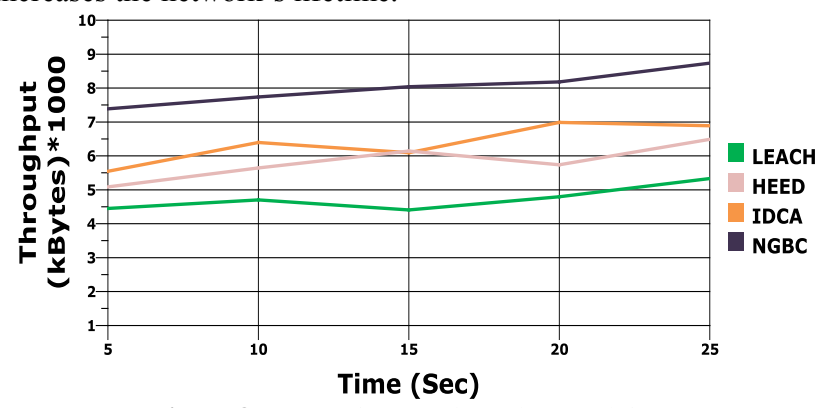

Figure 8: Comparison on throughput and time

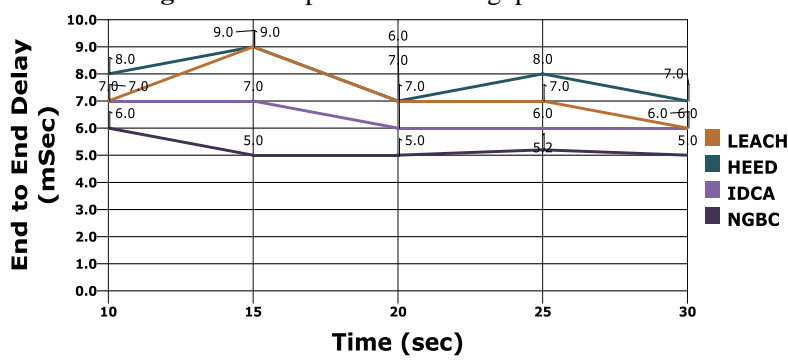

Figure 9: End to end delay Vs time

The above figure 9 depicted the throughput comparison with models. Generally, throughput is described as the ratio of total number of packets which is received by the destination from a sender at an instant the latest packet received at the receiver side evaluated in bytes/sec. From the graph, it is pointed out that the proposed work attains the higher through rate than others and proves its efficiency. End to End delay comparison is shown in the figure 8 . Typically, end to end delay denotes the average time taken by the data packet which is to be transmitted between the source node and the destination node across the whole network. In initial stage, the NGBC model involves $6.0 \mathrm{mSec}$ delay, whereas the other mechanism have slightly higher delay values.

As the time increases, the model constantly balancing the delay and it has to be noted as the model attaining minimum delay on packet transmission. Another important parameter on performance evaluation is Packet Delivery Ratio. In this experimental analysis, the reliability of the proposed work has been calculated by computing its packet delivery ratio (PDR) in correspondence to increase in time.

The packet delivery ratio comparison is shown in the following figure 9 . It is calculated by the ratio between the actual data packets received to the total data packets sent. The main property of a systematic clustering mechanism is to attain higher rate of PDR and it is evidenced from the graph.

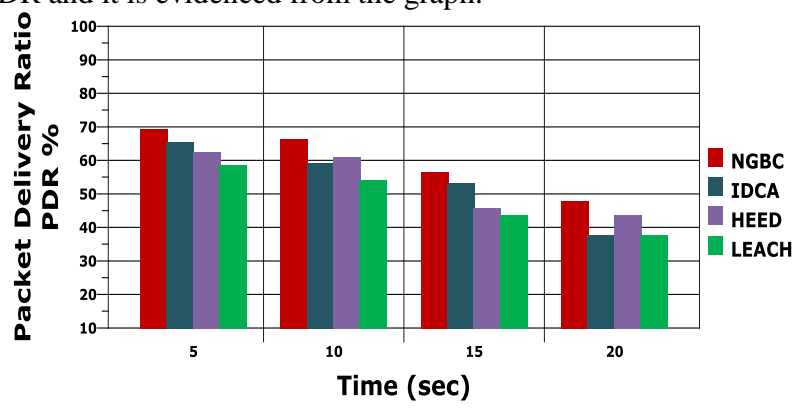

Figure 10: Comparison on packet delivery ratio

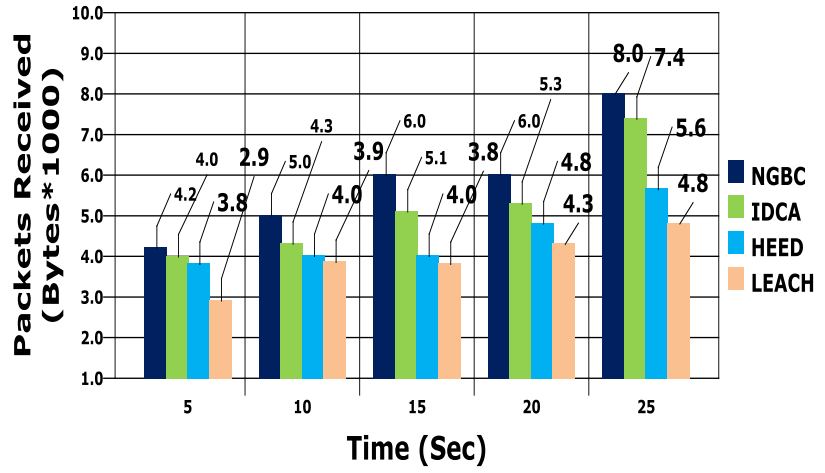

Figure 11: Number of packets received

The graph portrayed in figure 11 denotes the amount of packets received on BS in all methods that are compared over here. Initially, the packets receiving rate is low and later on it increases by time.

The proposed NGBC model attains higher rate of reception when compared with the existing LEACH, HEED and IDCA.

\section{Conclusion and future work}

On this paper, a systematic $\mathrm{CH}$ selection mechanism is proposed using Node-Grade Based Clustering (NGBC) Algorithm. After cluster formation, the $\mathrm{CH}$ selection is calculated in view on evaluating the remaining energy of candidate nodes and their Euclidean distance towards the BS. Moreover, for saving node from additional or unwanted energy consumption, Threshold Energy Rate (TER) is calculated for every node and the CHs are replaced by other nodes for each round when it is necessary. This results in reduction of the amount of energy consumption and also increases the network longevity. Through the performance evaluation, it is showed that the proposed algorithm surpasses the existing approaches like LEACH, HEED and IDCA. Further, it is found that using TER for cluster heads replacement enhancing the lifetime of the whole network. The results which are simulated clearly portrayed that the proposed NGBA algorithm achieving better throughput, lesser energy consumption, reduced end to end delay and also packet delivery ratio. The packet drop is also considerably reduced on compared to the previous techniques.

To be apparently said that this algorithm has some scope for future enhancement for providing better results with increase in network lifetime. The work could be extended by concerning some more challenging issues in WSN.

\section{References}

[1] Sajjanhar U \& Mitra P, "Distributive Energy Efficient Adaptive Clustering Protocol for Wireless Sensor Networks", International Conference on Mobile Data Management, (2007), pp.326 - 330.

[2] Zytoune Q, El. Aroussi M, Rziza M \& Aboutajdine D, "Stochastic Low Energy Adaptive Clustering Hierarchy”, ICGST-CNIR, Vol.8, No.1, (2008), pp.47-51

[3] Heinzelman WR, Chandrakasan A \& Balakrishnan H, "Energyefficient communication protocol for wireless micro sensor networks", IEEE Proceedings of the 33rd annual Hawaii international conference on System sciences, (2000), pp.1-10.

[4] Zytoune Q, Fakhri Y \& Aboutajdine D, "A Balanced Cost ClusterHeads Selection Algorithm for Wireless Sensor Networks", International Journal of Computer Science, Vol.4, No.1, (2009), pp.21-24.

[5] Du G, Shi Q, Tang Y \& Sun X, "A mixed non-uniform clustering algorithm for wireless sensor networks", IEEE $13^{\text {th }}$ International Conference Communication Technology (ICCT), (2011), pp.661665.

[6] Hong J, Kook J, Lee S, Kwon D and Yi S, "T-LEACH: the method of threshold-based cluster head replacement for wireless sensor networks", Information Systems Frontiers, Vol.11, No.5, (2011), pp.513-521. 
[7] Shu T, Krunz M \& Vrudhula S, "Power balanced coverage time optimization for clustered wireless sensor networks", 6th ACM International Symposium on Mobile Ad Hoc Networking and Computing, (2005), pp.111-120.

[8] Younis O, Krunz M \& Ramasubramanian S, "Node clustering in wireless sensor networks: recent developments and deployment challenges", IEEE Network, Vol.20 No.3, (2006), pp.20-25.

[9] Banerjee S \& Khuller S, "A clustering scheme for hierarchical control in multi-hop wireless networks", Twentieth Annual Joint Conference of the IEEE computer and communications societies, vol.2, (2001), pp.1028-1037.

[10] Fazackerley S, paeth A \& Lawrence R, "Cluster Head Selection Using RF Signal Strength", IEEE Canadian Conference on Electrical and Computer Engineering, (2009), pp.334 -338.

[11] Heinzelman W, Chandrakasan A \& Balakrishnan H, "An Application-Specific Protocol Architecture for Wireless Micrasensor Networks", IEEE Transactions on Wireless Communications, Vol.1, No.4, (2002), pp. 660-670.

[12] Younis O \& Fahmy S, "HEED: a hybrid, energy-efficient, distributed clustering approach for ad hoc sensor networks", IEEE Transactions on Mobile Computing, Vol.3, No.4, (2004), 366-379.

[13] Kumar N, Sandeep PB \& Mishra P, "U-LEACH: a novel routing protocol for heterogeneous wireless sensor networks", International Conference on Communication, Information \& Computing Technology (ICCICT), (2012)

[14] Xiangning F \& Yulin S, "Improvement on LEACH Protocol of Wireless Sensor Network", International Conference on Sensor Technologies and Applications, (2007), pp.260-264.

[15] Yassein M, Al-zou'bi A, Khamayseh Y \& Mardini W "Improvement on LEACH Protocol of Wireless Sensor Network (VLEACH)", JDCTA: International Journal of Digital Content Technology and its Applications, Vol.3, No.2, (2009), pp.132-136.

[16] Deepak Keynes JK \& Shalini Punithavathani D, "Using Integrated and Efficient Clustering Concept for Large Scale WSN", MiddleEast Journal of Scientific Research, Vol.25, No.3, (2017), pp.502-510.

[17] Dhanapal R \& Visalakshi P, "Efficient Clustering protocol based on Ant-Bee agent for Large Scale MANET", International Journal of Applied Engineering Research, Vol.10, (2015), pp. 349-361. 